\title{
TREND OF MAXIMAL INSPIRATORY PRESSURE IN MECHANICALLY VENTILATED PATIENTS: PREDICTORS
}

\author{
Pedro Caruso $^{\mathrm{a}, \mathrm{b}}$, Denise Simão Carnielib, Keila Harue Kagohara ${ }^{\mathrm{c}}$, Adriana
} Anciães $^{c}$, Jacqueline Santos Segarrab, Daniel Deheinzelin ${ }^{b}$

Caruso P, Carnieli DS, Kagohara KH, Anciães A, Segarra JS, Deheinzelin D. Trend of maximal inspiratory pressure in mechanically ventilated patients: predictors. Clinics. 2008;63(1):33-8.

INTRODUCTION: It is known that mechanical ventilation and many of its features may affect the evolution of inspiratory muscle strength during ventilation. However, this evolution has not been described, nor have its predictors been studied. In addition, a probable parallel between inspiratory and limb muscle strength evolution has not been investigated.

OBJECTIVE: To describe the variation over time of maximal inspiratory pressure during mechanical ventilation and its predictors. We also studied the possible relationship between the evolution of maximal inspiratory pressure and limb muscle strength.

METHODS: A prospective observational study was performed in consecutive patients submitted to mechanical ventilation for $>$ 72 hours. The maximal inspiratory pressure trend was evaluated by the linear regression of the daily maximal inspiratory pressure and a logistic regression analysis was used to look for independent maximal inspiratory pressure trend predictors. Limb muscle strength was evaluated using the Medical Research Council score.

RESULTS: One hundred and sixteen patients were studied, forty-four of whom (37.9\%) presented a decrease in maximal inspiratory pressure over time. The members of the group in which maximal inspiratory pressure decreased underwent deeper sedation, spent less time in pressure support ventilation and were extubated less frequently. The only independent predictor of the maximal inspiratory pressure trend was the level of sedation $(\mathrm{OR}=1.55,95 \%$ CI $1.003-2.408 ; \mathrm{p}=0.049)$. There was no relationship between the maximal inspiratory pressure trend and limb muscle strength.

CONCLUSIONS: Around forty percent of the mechanically ventilated patients had a decreased maximal inspiratory pressure during mechanical ventilation, which was independently associated with deeper levels of sedation. There was no relationship between the evolution of maximal inspiratory pressure and the muscular strength of the limb.

KEYWORDS: Respiratory Muscle. Mechanical Ventilation. Risk Factor. Muscular Paresis. Side Effects.

\section{INTRODUCTION}

The measurement of maximal inspiratory pressure (MIP), a useful index of respiratory muscle strength, is simple to perform and well tolerated by patients. ${ }^{1}$ We have

${ }^{\text {a }}$ Respiratory Intensive Care Unit, Pulmonary Division, Hospital das Clínicas, Faculdade de Medicina da Universidade de São Paulo - São Paulo/SP, Brazil.

${ }^{b}$ Intensive Care Unit, Hospital A. C. Camargo - São Paulo/SP, Brazil. cPhysiotherapy Division, Hospital das Clínicas, Faculdade de Medicina da Universidade de São Paulo - São Paulo/SP, Brazil. pedrocaruso@uol.com.br

Received for publication on September 28, 2007. Accepted for publication on October 15, 2007. shown that the use of a unidirectional valve reduces the variation in measurement due to volitional causes, therefore creating a more reliable and reproducible measurement over time. ${ }^{2}$ In the intensive care unit, MIP may be used as an index for weaning patients from mechanical ventilation, ${ }^{3}$ although it may not be as accurate as other indices. ${ }^{4}$ It is also used as a diagnostic tool, particularly when muscular diseases are suspected to be the cause of respiratory failure. ${ }^{5}$ In this setting, MIP can be impaired due to respiratory muscle atrophy, dysfunction caused by mechanical ventilation, critical illness myopathy or the use of steroids. ${ }^{6-8}$ Moreover, patients with systemic inflammatory response syndrome (SIRS), a common finding in the intensive care 
unit (ICU), tend to have a lower MIP than patients without SIRS. ${ }^{9}$

It is known that mechanical ventilation and many of its features can affect the evolution of inspiratory muscle strength during ventilation. ${ }^{10}$ However, this evolution has not been described, and its predictors have not been studied. Recently, it was shown that in critically ill mechanically ventilated patients submitted to endurance training, the MIP improved over time ${ }^{11}$ for reasons that are not fully established, while the MIP deteriorated in patients without such training. As such, the primary objective of the present study was to verify how the MIP varies over time in critically ill mechanically ventilated patients and to verify possible predictors of these variations. As a secondary objective, we evaluated the relationship between the MIP trend during mechanical ventilation and limb muscle strength at the end of the mechanical ventilation period. This objective was elected because the probable relationship between the respiratory and limb muscular systems involved in long term mechanically ventilated patients has not yet been elucidated. ${ }^{12}$

\section{METHODS}

Since this was a prospective observational study, apart from MIP measurements, there was no intervention in any planned treatment. The study was performed in three surgical-clinical ICUs, containing 23, 4, and 6 beds. The inclusion criterion was consecutive adults patients for whom prolonged mechanical ventilation ( $>72$ hours) was foreseen. The exclusion criteria were flail chest, known coronary artery disease, alveolar hemorrhage, mean arterial pressure under $70 \mathrm{~mm} \mathrm{Hg}$ after volume resuscitation or previous peripheral neuromuscular disease.

MIP was measured daily, always in the morning, using a unidirectional valve as previously described. ${ }^{2}$ Briefly, one side of an aneroid manometer (Record; São Paulo-Brazil) capable of registering pressures up to $150 \mathrm{~cm} \mathrm{H}_{2} \mathrm{O}$ was attached to the patient's orotracheal or tracheostomy tube, and the other side was attached to a unidirectional, lowresistance valve that only allowed expiration. MIP was measured by connecting patients to the manometer during a $20 \mathrm{~s}$ period and recording the maximal value of three maneuvers.

To assess the MIP trend of each patient, a linear regression of the daily measurements of the MIP was performed. A negative coefficient value meant a trend toward a decrease in the MIP, and a positive coefficient indicated an MIP increase.

The patients' demographic data, as well as the main reason for mechanical ventilation, were collected upon admis- sion into the study. The following seven variables that could influence the MIP trend were recorded daily: 1 . Sedation, which was at the discretion of the attending physician, but mostly consisted of intravenous midazolam and fentanyl. The three ICUs use a daily interruption sedation protocol. Sedation was checked 3 times a day using the Ramsay scale $^{13}$ and the mean value for each patient was used for statistical purposes; 2. Use of a neuromuscular blocking agent prescribed in the previous $24 \mathrm{~h}$ at the time of MIP measurement, but more than 6 hours before the MIP measurement; 3 . The mode of the mechanical ventilation (controlled/assisted mechanical ventilation or pressure support ventilation) applied for the majority of the day; 4. Fasting, when no enteral feeding or parenteral solutions other than glucose solution had been given to the patient in the previous $24 \mathrm{~h} ; 5$. The presence of SIRS based on established criteria $;{ }^{14} 6$. The use of corticosteroids and 7 . The mean value of the capillary glucose measurements.

Immediately after extubation, given that awakening and comprehension were confirmed, limb muscle strength was evaluated using a simple bedside muscle strength score: the Medical Research Council (MRC) score. Patients with an MRC score less than 48 were considered to have ICU paresis. ${ }^{15,16}$

If reintubation was not required within 48 hours, extubation success was declared.

This protocol was conducted in accordance with the ethical standards of our institution's committees on human experimentation.

\section{Statistical analysis}

Because the distribution of most of the data was not normal, comparison between patients with an increased or decreased MIP was performed using the Chi square or Fischer exact tests for categorical variables and MannWhitney analysis for continuous variables. In order to identify the predictors of the MIP trend, we performed a univariate regression analysis to identify variables yielding $\mathrm{p}<0.20$, which were entered into a logistic regression. In order to avoid logistic regression model overfitting, we did not use stepwise procedures and we chose the predictor variables based on a univariate regression analysis yielding $\mathrm{p}<0.20$ or clinical relevance. Ten independent variables (age, Apache $\mathrm{II}^{17}$ at admission, ventilation cause, mean Ramsay scale, mean capillary glucose, $\%$ of days with pressure support ventilation, \% days with neuromuscular blocking agents, $\%$ days with steroids, $\%$ days with systemic inflammatory response syndrome and $\%$ days of fasting) were selected for the logistic regression model

Data are expressed as median, minimum and maximum. 


\section{RESULTS}

One hundred and sixteen consecutive patients entered the protocol, and their characteristics are summarized in table 1 . Seventy patients were extubated during the study (60.3\%), 32 died (27.6\%) and 14 had their life support withdrawn (12.1\%); eighteen of the extubated patients were reintubated $(25.7 \%)$ before 48 hours. Most patients presented an abnormally low MIP on the first and last days of mechanical ventilation (Figure 1).

Forty-four patients disclosed a negative coefficient (MIP-), while in the remaining 72 , a positive coefficient was observed, characterizing improvement of MIP overtime (MIP+) (Table 1 and Figure 1).

Patients in the MIP- group received heavier sedation, spent more time with pressure support ventilation and were extubated less frequently. Also, there was a trend towards higher levels of mean capillary glucose in the patients that decreased their MIP during mechanical ventilation (Table $1)$.

The logistic regression analysis showed that the only independent predictor for MIP decrease during mechanical ventilation was the level of sedation (mean Ramsay score) $(\mathrm{OR}=1.55,95 \%$ CI $1.003-2.408 ; \mathrm{p}=0.049)$.

We did not find any relationship between the MIP trend and limb muscle strength at extubation (Table 2).
Daily Evolution of Maximal Inspiratory Pressure

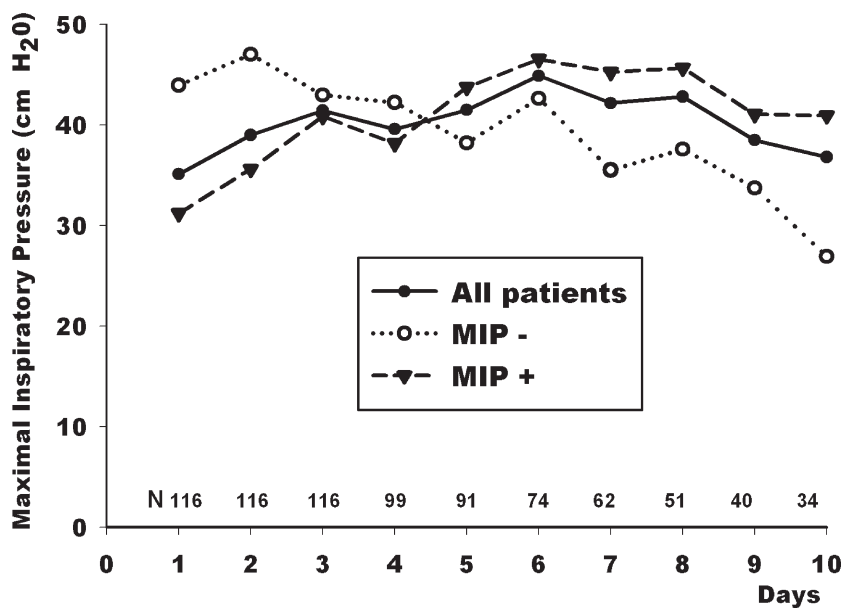

Figure 1 - Daily evolution of maximal inspiratory pressure. MIP = Maximal inspiratory pressure. MIP+ = group of patients with increased MIP during mechanical ventilation. MIP- = group of patients with decreased MIP during mechanical ventilation. The numbers above the $\mathrm{X}$ axis are the number of patients in the protocol.

\section{DISCUSSION}

In this observational study, most of the patients were found to present low values of maximal inspiratory pressure at the beginning and end of the mechanical ventila-

Table1 - Demographic characteristics and a comparison of patients with a maximal inspiratory pressure increase (MIP+) and decrease (MIP-) over time.

\begin{tabular}{|c|c|c|c|c|}
\hline & All Patients & $\mathrm{MIP}+$ & MIP- & $\mathrm{P}$ \\
\hline $\mathrm{N}(\%)$ & 116 & $72(62.1)$ & $44(37.9)$ & \\
\hline Age (years) & $66(15-90)$ & $67(22-88)$ & $58.5(15-90)$ & 0.44 \\
\hline Male (\%) & $66(56.9)$ & $41(56.9)$ & $25(56.8)$ & 1.0 \\
\hline Apache II at admission & $19(6-34)$ & $21(6-34)$ & $14.5(10-27)$ & 0.08 \\
\hline Ventilation Cause(\%) & & & & 0.21 \\
\hline Acute respiratory failure & $69(59.5)$ & $39(54.2)$ & $30(68.2)$ & \\
\hline Decreased consciousness & $30(25.9)$ & $19(26.4)$ & $11(25.0)$ & \\
\hline Hemodynamic Instability & $13(11.2)$ & $10(13.9)$ & $3(6.8)$ & \\
\hline Others & $4(3.4)$ & $4(5.5)$ & 0 & \\
\hline Days in protocol & $8(3-26)$ & $7.5(3-26)$ & $8(3-8)$ & 0.97 \\
\hline Extubated (\%) & $70(60.3)$ & $49(68 \%)$ & $21(47.7 \%)$ & 0.034 \\
\hline Weaning failure $(\%)$ & $18(25.7)$ & $14(28.6)$ & $4(19)$ & 0.55 \\
\hline Ramsay scale, mean & $5(0.4-6)$ & $4.8(0.4-6)$ & $5.5(3.1-6)$ & 0.028 \\
\hline Mean capillary glucose & $141(99-260)$ & $139(99-210)$ & $159(99-260)$ & 0.08 \\
\hline \% days in PSV & $40(0-100)$ & $45.5(0-100)$ & $27.5(0-100)$ & 0.044 \\
\hline$\%$ days with sedation & $90.2(0-100)$ & $86(0-100)$ & $100(0-100)$ & 0.81 \\
\hline$\%$ days with NMB agents & $0(0-25)$ & $0(0-25)$ & $0(0-25)$ & 0.72 \\
\hline$\%$ days with steroids & $83.3(0-100)$ & $84.9(0-100)$ & $80.9(0-100)$ & 0.59 \\
\hline$\%$ days with SIRS & $93.6(14-100)$ & $100(14-100)$ & $83.3(14-100)$ & 0.27 \\
\hline$\%$ days of fasting & $16.6(0-100)$ & $14.6(0-100)$ & $20(0-100)$ & 0.18 \\
\hline
\end{tabular}

MIP = Maximal inspiratory pressure. MIP+ = group of patients with increased MIP during mechanical ventilation. MIP- = group of patients with

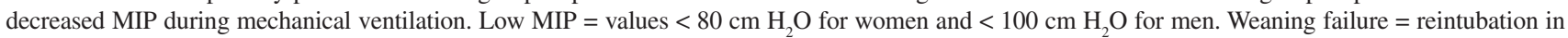
less than 48 hours. PSV = Pressure support ventilation. NMB agents $=$ Neuromuscular blocking agents. SIRS $=$ Systemic Inflammatory Response Syndrome. Values are expressed as median values with maximum and minimum values in parentheses. 
Table 2 - Relationship between MIP trend and limb muscle strength.

\begin{tabular}{lcccc}
\hline & All Patients & MIP+ & MIP- & $\mathrm{p}$ \\
\hline $\mathrm{N}(\%)$ & 69 & $48(69.6)$ & $21(30.4)$ & \\
$\mathrm{MRC}$ score & $46(0-60)$ & $45.5(0-60)$ & $48(0-60)$ & 0.72 \\
$\%$ patients with ICU paresis & $36(52.2)$ & $26(54.2)$ & $10(47.6)$ & 0.79 \\
\hline
\end{tabular}

MIP = Maximal inspiratory pressure. MIP+ = group of patients with increased MIP during mechanical ventilation. MIP- = group of patients with decreased MIP during mechanical ventilation. MRC score = Medical Research Council score. ICU paresis = patients with an MRC score less than 48.

tion period, and around $40 \%$ had a decreasing MIP during mechanical ventilation. The univariate analysis determined that patients with decreased MIP were less frequently extubated, had higher levels of sedation and were under pressure support ventilation over less of the mechanical ventilation period. Also, MIP- patients tended to have higher levels of capillary glucose, reflecting worse glucose control. In the multivariate analysis, the level of sedation reflected by the mean Ramsay scale value was an independent predictor of the MIP trend. We did not find any relation between MIP trend and limb strength (ICU paresis).

The low levels of MIP at entry in this study and the low levels even on the day of extubation are in accordance with our ${ }^{11}$ and other previous observations. ${ }^{18}$ This is probably due to the reduction in muscle contractility and nerve conduction of critically ill patients, ${ }^{19}$ the recently described detrimental effect of mechanical ventilation on the contractile properties of the diaphragm ${ }^{10}$ or an effect of the sedatives on MIP. However, it must be noted that on the day of extubation, the mean MIP remained below the normal range in the extubated patients. At this point, the sedative effect was minimal and patients were more cooperative.

The use of more periods of pressure support ventilation was found to be a determinant of MIP improvement over time. Possible explanations for this may be found in the evidence of diaphragm dysfunction in animals submitted to fully controlled modes of mechanical ventilation, ${ }^{10}$ and the capability of even short periods of assisted ventilation modes such as pressure support to prevent the loss of the diaphragmatic force-generating capacity observed with controlled modes of ventilation..$^{20}$ Additionally, in weaning trials, the use of pressure support was associated with a higher percentage of successful spontaneous breathing when compared to T-tubes ${ }^{21}$ or intermittent mandatory ventilation. ${ }^{22}$

An independent predictor of the MIP trend was the level of sedation. Our population was sedated most of the time while under mechanical ventilation, which is in accordance with recent data showing that the use of sedatives is very common and is associated with a longer duration of mechanical ventilation in patients like ours. ${ }^{23}$ There are very few randomized studies to define the best regimen of sedation in intensive care, ${ }^{24}$ and the best regimen for each mode of ventilation has yet to be defined..$^{25}$ Of note, recent experimental data showed that midazolam causes diaphragm dysfunction in a dose-related manner, ${ }^{26}$ which may explain why more profound sedation is associated with a decrease in respiratory muscle strength. Interestingly, in a recent study of daily interruption of sedative infusion, the total dose of midazolam was reduced by almost half, and the patients spent 2 days less under mechanical ventilation. ${ }^{27}$ Another study associated prolonged mechanical ventilation with continuous IV sedation..$^{28}$ Taken together, these results suggest that inspiratory muscle strength impairment may occur as a result of the sedation strategies utilized in most ICUs.

We can hypothesize that MIP evolution during mechanical ventilation either depends on the interventions performed (sedation, use of fully controlled mechanical ventilation modes, glucose control, etc.) or is independent of them and is associated with the clinical condition at admission. In our study, we have an indication that the interventions during mechanical ventilation may be more important than the clinical condition at admission because in the MIP+ group, there was a trend toward a higher APACHE II score at admission ( $21 \times 14.5: \mathrm{p}=0.08)$, but the group had an increase in MIP during ventilation instead of a decrease.

We hypothesized a direct relationship between the inspiratory muscle strength trend and the limb muscle strength at the end of the mechanical ventilation period, expecting more intense or frequent limb paresis in the group of patients with decreased MIP during mechanical ventilation. This is a feasible relationship because both are skeletal muscles submitted to the same insults, and there is an association between development of ICU paresis and longer mechanical ventilation ${ }^{16}$. In our study, however, we did not find any relation between the MIP trend and qualitative (percentage of patients with limb paresis) or quantitative (MRC score) alteration of limb muscle strength.

One limitation of the study was the use of MIP to measure inspiratory muscle strength. MIP is a volitional method, so it can be affected by sedation, lack of motivation and variations in the level of consciousness. In order to counteract these factors, we measured the MIP with a unidirec- 
tional valve. This method captures the physiologic response of increasing respiratory drive after a previous inefficient inspiration, thereby demanding less patient collaboration; this method has been shown to be reasonably reproducible ${ }^{2}$. Another limitation is the fact that we placed patients with all possible causes of respiratory failure into an acute respiratory failure group and did not analyze the sub-groups inside it, such as chronic obstructive pulmonary disease or pneumonia. This was done because the number of patients in the sub-groups would have been insufficient to make statistical conclusions. However, it remains possible that the various causes of respiratory failure have an impact on the MIP evolution.

In conclusion, around forty percent of mechanically ventilated patients have a decrease in maximal inspiratory pressure during mechanical ventilation. Deeper levels of sedation are also associated with a decrease in maximal inspiratory pressure during mechanical ventilation. There was, however, no relationship between the evolution of maximal inspiratory pressure and the limb muscular strength at the end of the ventilatory period.

\section{REFERENCES}

1. ATS/ERS Statement on respiratory muscle testing. Am J Respir Crit Care Med. 2002;166:518-624.

2. Caruso P, Friedrich C, Denari SD, Ruiz SA, Deheinzelin D. The unidirectional valve is the best method to determine maximal inspiratory pressure during weaning. Chest. 1999;115:1096-101.

3. Sahn SA, Lakshminarayan S. Bedside criteria for discontinuation of mechanical ventilation. Chest. 1973;63:1002-5.

4. Yang KL, Tobin MJ. A prospective study of indexes predicting the outcome of trials of weaning from mechanical ventilation. $\mathrm{N}$ Engl $\mathrm{J}$ Med. 1991;324:1445-50.

5. Black LF, Hyatt RE. Maximal static respiratory pressures in generalized neuromuscular disease. Am Rev Respir Dis. 1971;103:641-50.

6. Le Bourdelles G, Viires N, Boczkowski J, Seta N, Pavlovic D, Aubier M. Effects of mechanical ventilation on diaphragmatic contractile properties in rats. Am J Respir Crit Care Med. 1994;149:1539-44.

7. van Balkom RH, van der Heijden HF, van Herwaarden CL, Dekhuijzen PN. Corticosteroid-induced myopathy of the respiratory muscles. Neth J Med. 1994;45:114-22.

8. Latronico N, Fenzi F, Recupero D, Guarneri B, Tomelleri G, Tonin P, et al. Critical illness myopathy and neuropathy. Lancet. 1996;347:157982.

9. Amoateng-Adjepong Y, Jacob BK, Ahmad M, Manthous CA. The effect of sepsis on breathing pattern and weaning outcomes in patients recovering from respiratory failure. Chest. 1997;112:472-7.
10. Vassilakopoulos T, Petrof BJ. Ventilator-induced diaphragmatic dysfunction. Am J Respir Crit Care Med. 2004;169:336-41.

11. Caruso P, Denari SD, Ruiz SA, Bernal KG, Manfrin GM, Friedrich C, et al. Inspiratory muscle training is ineffective in mechanically ventilated critically ill patients. Clinics. 2005;60:479-84.

12. De Jonghe B, Sharshar T, Hopkinson N, Outin H. Paresis following mechanical ventilation. Curr Opin Crit Care. 2004;10:47-52.

13. Ramsay MA, Savege TM, Simpson BR, Goodwin R. Controlled sedation with alphaxalone-alphadolone. Br Med J. 1974;2:656-9.

14. American College of Chest Physicians/Society of Critical Care Medicine Consensus Conference:definitions for sepsis and organ failure and guidelines for the use of innovative therapies in sepsis. Crit Care Med. 1992;20:864-74.

15. Kleyweg RP, van der Meche FG, Schmitz PI. Interobserver agreement in the assessment of muscle strength and functional abilities in GuillainBarre syndrome. Muscle Nerve. 1991;14:1103-9.

16. De Jonghe B, Sharshar T, Lefaucheur JP, Authier FJ, Durand-Zaleski I, Boussarsar M, et al. Paresis acquired in the intensive care unit:a prospective multicenter study. Jama. 2002;288:2859-67.

17. Knaus WA, Draper EA, Wagner DP, Zimmerman JE. APACHE II:a severity of disease classification system. Crit Care Med. 1985;13:81829.

18. Laghi F, Cattapan SE, Jubran A, Parthasarathy S, Warshawsky P, Choi YS, et al. Is weaning failure caused by low-frequency fatigue of the diaphragm? Am J Respir Crit Care Med. 2003;167:120-7. 
19. Wagenmakers AJ. Muscle function in critically ill patients. Clin Nutr. 2001;20:451-4.

20. Sassoon CS, Zhu E, Caiozzo VJ. Assist-control mechanical ventilation attenuates ventilator-induced diaphragmatic dysfunction. Am J Respir Crit Care Med. 2004;170:626-32.

21. Esteban A, Alia I, Gordo F, Fernandez R, Solsona JF, Vallverdu I, et al. Extubation outcome after spontaneous breathing trials with T-tube or pressure support ventilation. The Spanish Lung Failure Collaborative Group. Am J Respir Crit Care Med. 1997;156:459-65.

22. Brochard L, Rauss A, Benito S, Conti G, Mancebo J, Rekik N, et al. Comparison of three methods of gradual withdrawal from ventilatory support during weaning from mechanical ventilation. Am J Respir Crit Care Med. 1994;150:896-903.

23. Arroliga A, Frutos-Vivar F, Hall J, Esteban A, Apezteguia C, Soto L, et al. Use of sedatives and neuromuscular blockers in a cohort of patients receiving mechanical ventilation. Chest. 2005;128:496-506.
24. Ostermann ME, Keenan SP, Seiferling RA, Sibbald WJ. Sedation in the intensive care unit:a systematic review. Jama. 2000;283:1451-9.

25. Carrasco G. Instruments for monitoring intensive care unit sedation. Crit Care. 2000;4:217-25.

26. Fujii Y, Uemura A, Toyooka H. Midazolam-induced muscle dysfunction and its recovery in fatigued diaphragm in dogs. Anesth Analg. 2003;97:755-8.

27. Kress JP, Pohlman AS, O'Connor MF, Hall JB. Daily interruption of sedative infusions in critically ill patients undergoing mechanical ventilation. N Engl J Med. 2000;342:1471-7.

28. Kollef MH, Levy NT, Ahrens TS, Schaiff R, Prentice D, Sherman G. The use of continuous i.v. sedation is associated with prolongation of mechanical ventilation. Chest. 1998;114:541-8. 